\title{
"Os Trigonum" y "Proceso de Stieda" en el Síndrome del Impacto Posterior del Tobillo
}

\author{
"Os Trigonum" and "Stieda Process" in Posterior Ankle Impingement Syndrome
}

\author{
Henrique Ayres de Vasconcellos*; Mey Lie Tan Maia de Holanda Cavalcante ${ }^{*, * *}$; \\ Mario Manoel Parreira Fortes**; Patrícia Paula Neves* \& Ana Carolina Kopke Rocha*
}

\begin{abstract}
VASCONCELlOS, H. A.; CAVAlCANTE, M. L. T M. H.; FORTES, M. M. P.; NEVES, P. P. \& ROCHA, A. C. K. "Os trigonum” y "Proceso de Stieda" en el síndrome del impacto posterior del tobillo. Int. J. Morphol., 31(4):1223-1226, 2013.

RESUMEN: El Os trigonum (OT) y el Proceso de Stieda (PS), corresponden a la osificación separada o extensiva del tubérculo lateral de la cara posterior del talus (TA), respectivamente. Son indicados como factores causales del Síndrome del Impacto Posterior del Tobillo (SIPT), disturbio, agudo o crónico, con dolor en la región posterior del tobillo a los movimientos del pie. El objetivo fue analizar la importancia morfológica de la presencia del OT y/o PS y su posible relación con el SIPT. Fueron utilizados 80 TA secos. La morfometría del TA y del Tubérculo Lateral del TA (TL) fueron calculadas y expresas como: LTA = largo total del TA; ATA = ancho total del TA; LTL/ LPS = largo total de TL/PS; ETL = espesor total de TL/PS. Los análisis mostraron valores de LTA: 55,22 ( $\pm 4,16)$ mm; ATA: 39,51 $( \pm 2,46) \mathrm{mm}$; LTL/LPS: $8,06 \mathrm{~mm}( \pm 2,14)$ y ETL: $8,46( \pm 1,01) \mathrm{mm}$. Nuestros resultados ofrecen informaciones no encontradas en la literatura especializada y ayudan en el diagnóstico y tratamiento del SIPT.
\end{abstract}

PALABRAS ClAVE: Os Trigonum; Proceso de Stieda; Talus; Anatomía del Pie.

\section{INTRODUCCIÓN}

El "Síndrome del Impacto Posterior del Tobillo "(SIPT), "Síndrome Os trigonum" o "Síndrome Compresiva Tibiotalar Posterior", es el disturbio clínico, agudo o crónico, caracterizado por dolor en la región posterior del tobillo a los movimientos del pie, principalmente en la flexión plantar (Lima et al., 2010; Chiereghin et al., 2011).

El tubérculo lateral (TL) del talus (TA) puede presentarse modificado originando el "Os Trigonum" (OT) o el "«Proceso de Stieda»" (PS) (Fig. 1). Tenosinovitis del músculo flexor largo del hallux, osteocondritis de tobillo, enfermedad de la articulación subtalar, fracturas, presencia de OT o PS pueden llevar a la compresión de los tejidos cercanos siendo señalados como causa de la SIPT (Bergman \& Thompson, 1984; Chiereghin et al.).

Anatomia del Talus. El proceso posterior del TA presenta un surco para el tendón del músculo flexor largo del hallux, y 2 tubérculos, uno lateral (TL) y otro medial (TM). El TL se origina de un punto de osificación secundario que se une al cuerpo del TA, entre los 7 y 13 años de edad; esta fusión dura 1 año. Flexiones plantares forzadas en la adolescencia, osificaciones parciales o totales expuestas a fracturas sin consolidación, pueden determinar la aparición de un hueso accesorio, el "Os Trigonum" (OT), descrito en 14 a 25\% de los huesos adultos (Fig. 2). Las primeras descripciones del OT son atribuidas a los auores Rosenmuller en 1804, Schwegel en 1858, Gruber en 1864, y más tarde por Bardeleben lo denominó "Hueso Trígono"/"Os Trigonum"/ "Intermedio del Tarso". Stieda nombró un proceso "«Proceso de Stieda»" (PS) al estiramiento del TL del TA, habiendo referencias a la presencia bilateral del TL/PS (Poirier \& Charpy, 1911; Testut \& Latarjet, 1959; Llorca, 1967; Adams \& Hamblen, 1994; Lima et al.; Chiereghin et al.; Moore et al., 2011).

El objetivo de este estudio fue revisar la morfología del proceso posterior del TA; sugerir una definición para TL, OT, PS, y analizar la posible participación, de esas estructuras en la SIPT.

\footnotetext{
* Laboratorio de Anatomia, Facultad de Medicina de Petrópolis/FASE, Rio de Janeiro, Brasil.

** Laboratorio de Anatomia, UNIFESO, Teresópolis, Rio de Janeiro, Brasil.
} 


\section{MATERIAL Y MÉTODO}

Utilizamos 80 TA secos, 74 izquierdos $(92,5 \%)$ y 6 derechos (7,5\%), 5 articulados $(6,25 \%)$ y 75 no articulados $(93,75 \%)$, pertenecientes a los acervos de los Laboratorios de los Departamentos de Anatomía de las FMP/FASE/ Petrópolis/RJ/Brasil y UNIFESO/Teresópolis/RJ/Brasil.

Después de una limpieza rigorosa realizamos la morfometría ósea que consistía en tomar medidas, en sus mayores extensiones, en línea recta, con paquímetro (precisión milimétrica), siendo señaladas las medias aritméticas de 3 medidas consecutivas, obtenidas por un único examinador (Figa. 1 y 3 ).

1. Talus: $\mathrm{LTA}=$ largo del TA; ATA $=$ ancho del TA.

2. Tubérculo Lateral / «Proceso de Stieda»: LTL / LPS = largo del TL / PS; ETL / EPS = espesor del TL / PS.

\section{RESULTADOS}

Se obtuvieron los siguientes valores medios en milímetros:

I) Talus

LTA $=55,22( \pm 3,64)$. Mayor medida $=63,96$ y menor medida $=46,06$.

$\mathrm{ATA}=39,51( \pm 2,46)$. Mayor medida $=45,16$ y menor medida $=33,53$.

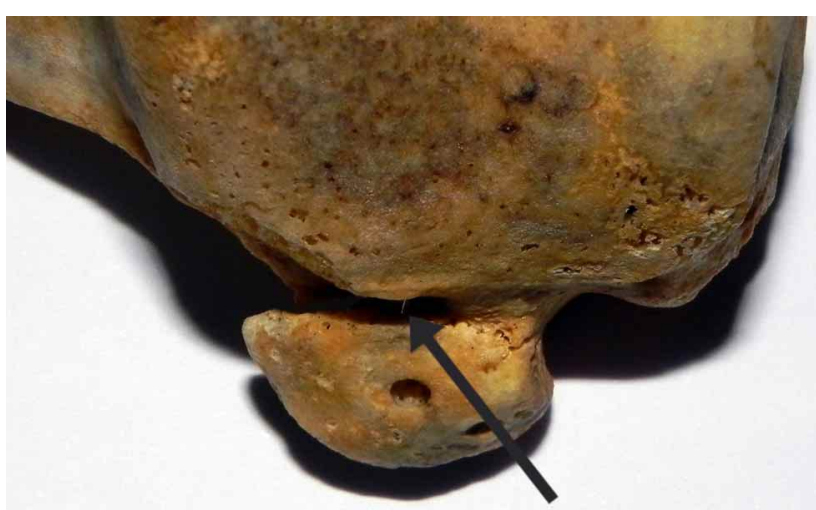

Fig. 2. Osificación parcial del tubérculo lateral del talus (flecha negra).

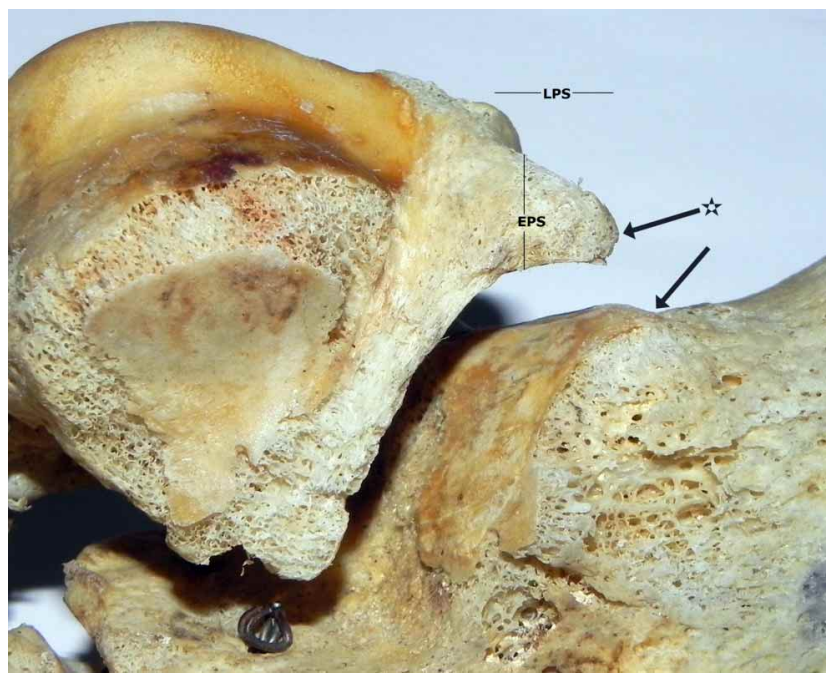

Fig. 3. Estrella + flecha negra $=«$ Proceso de Stieda $»$; flecha negra $=$ Cara articular del calcáneo; LPS= Largo del «Proceso de Stieda»; EPS $=$ Grosor del «proceso de Stieda».
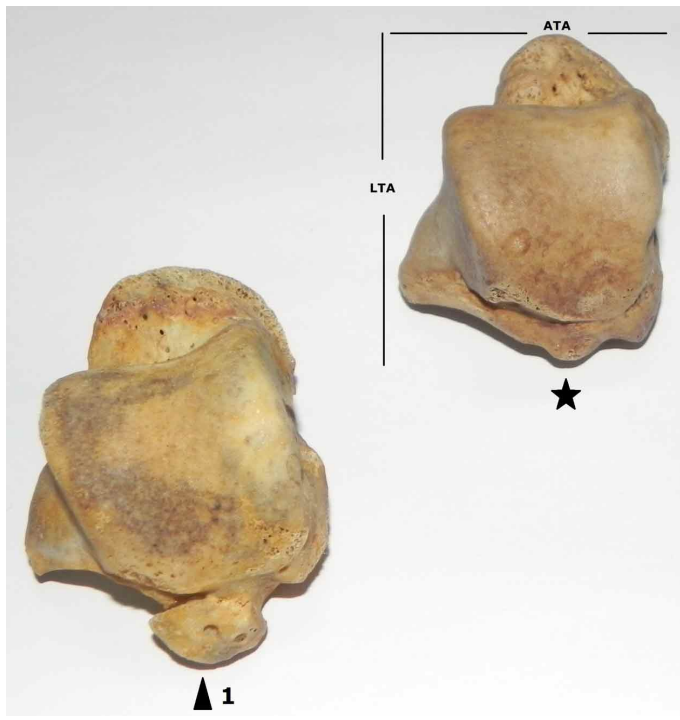

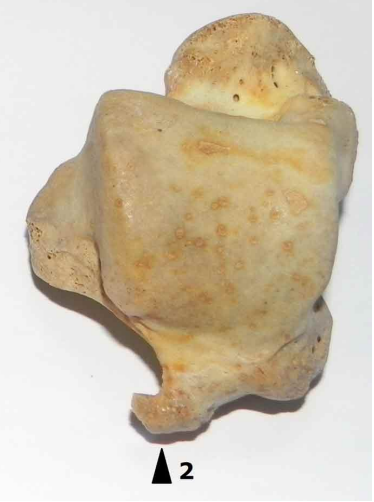

Fig. 1. Estrella negra= Tubérculo lateral del talus; ATA= Ancho del talus; LTA: Largo del talus; $1=$ «Proceso de Stieda» parcialmente fusionado, $2=\ll$ Proceso de Stieda $»$ con desvío lateral. 
II) Tuberculo lateral / «Proceso de Stieda»

$\mathrm{LTL}=8,06( \pm 1,71)$. Mayor medida $=13,03$ y menor medida $=2,53$ (Fig. 4).

$\mathrm{ETL}=8,46( \pm 1,01)$. Mayor medida $=12,13$ y menor medida $=6,0$.

En los 5 esqueletos articulados la cara articular del hueso calcáneo acompañó el alargamiento del TL / PS (Fig. 3).

\section{DISCUSIÓN}

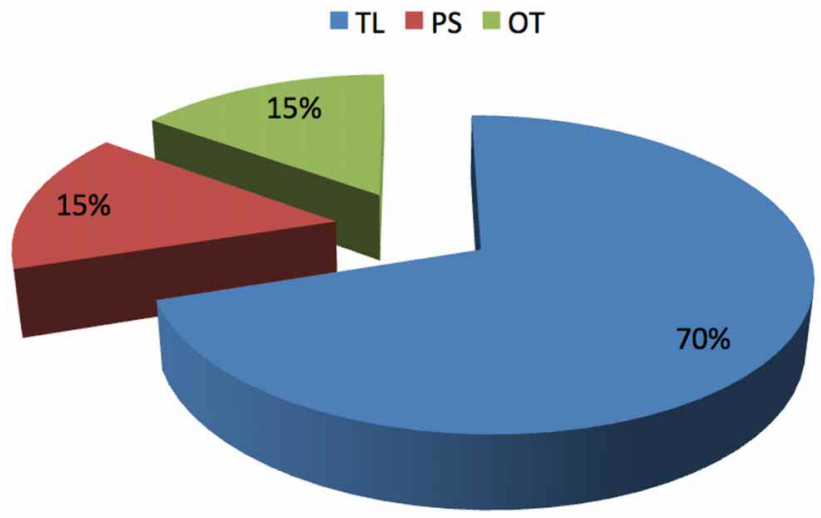

Fig. 4. Os Trigonum: Tubérculo Lateral/Proceso de Stieda (OT:LTL) $<5 \mathrm{~mm}$. «Proceso de Stieda»: Tubérculo lateral/ «Proceso de Stieda» (PS: LTL) > $10 \mathrm{~mm}$. Tubérculo lateral:Tubérculo lateral $/ \ll$ Proceso de Stieda» (TL: LTL) $=5-10 \mathrm{~mm}$.

Poirier \& Charpy establecieron valores de 5 a $10 \mathrm{~mm}$ para el OT, que después de la fusión al cuerpo del TA, formarían el TL. Jabonlay (op. cit. Poirier \& Charpy) reportan en un estudio de 111 TA el OT en 20 casos (18\%), de los cuales, 15 (13\%) medían $10 \mathrm{~mm}$ de largo. LLorca describe el OT o Talus accesorio, que puede alcanzar hasta $15 \mathrm{~mm}$ de diámetro, y se encontró dividido en dos (Os Trigonum Bipartitum). Estos autores no describen los parámetros utilizados para la obtención de las medidas. El análisis de los datos reveló que los valores citados son similares a los encontrados para la LTL.

Para Xhardez (2001), Thompson (2004) y Chiereghin et al. el OT se osifica entre los 7 y 13 años de edad combinándose al TA para formar la PS. Xhardez y Chiereghin et al. refieren que el OT es de causa común de la SIPT, así como la tenosinovitis del músculo flexor largo del hallux y la enfermedad de la articulación subtalar. Observamos en los huesos calcáneos articulados, el alargamiento de sus superficies articulares para el PS, lo que puede justificar tanto la tenosinovitis como la enfermedad articular.
No encontramos en la literatura clásica y/o especializada, la aclaración necesaria sobre el significado de las estructuras estudiadas y, por esta razón, sugerimos las siguientes definiciones: Os Trigonum, es el punto secundario de osificación que no se solda al TA. De lo contrario se formarán el TL. Tubérculo lateral, es el tubérculo existente en la región posterior y lateral del TA con valores entre 5 a 10 mm de largo. «Proceso de Stieda» es una extensión de la TL cuando alcanza valores superiores a $10 \mathrm{~mm}$.

\section{CONCLUSIÓN}

Nuestros datos, agregados a los citados por la literatura, aumentan la calidad descriptiva de estos accidentes anatómicos. Las definiciones propuestas para "TL", "OT", "PS", y la identificación del alargamiento de la superficie articular del calcáneo, son informaciones significativas para la determinación de los factores causadores de la SIPT.

\section{AGRADECIMIENTOS}

Agradecemos a la Profesora Brenda Tan Maia por la versión en Español del texto, a João Amaral Galvão por la documentación fotográfica.

VASCONCELLOS, H. A.; CAVALCANTE, M. L. T M. H.; FORTES, M. M. P.; NEVES, P. P. \& ROCHA, A. C. K. " $O s$ Trigonum" and "Stieda Process" in posterior ankle impingement syndrome.Int. J. Morphol., 31(4):1223-1226, 2013.

SUMMARY: Os Trigonum (OT) and Stieda Process (SP), separate or extensive ossification of the lateral tubercule of posterior face of Talus (TA), respectively, are indicative of causal factors of the Posterior Impingement Syndrome of The Ankle (PISA) disturbance, sharp or chronic pain, with pain in the posterior region of the ankle caused by foot movements. The objective of this study was to analyze the morphological importance of the presence of OT and or SP and the possible relationship with PISA for which we used 80 dry TA. The morphometry of TA and Lateral Tubercule of TA (LT) were calculated and expressed as mean: LTA= total length of TA; WTA= total width of TA; LLT/LPS= total length of LT/SP; TLT $=$ total thickness of LT/SP. The measures were LTA: $55.22( \pm 4.16) \mathrm{mm}$; WTA: $39.51( \pm 2.46) \mathrm{mm}$; LLT/LPS: 8.06 $( \pm 2.14) \mathrm{mm}$; TLT: $8.46( \pm 1.01) \mathrm{mm}$. Our results provide useful information not found in the specialized literature and are of assistance in the diagnosis and treatment of PISA.

KEY WORDS: Os Trigonum; Stieda Process; Talus; Foot Anatomy. 
VASCONCEllos, H. A.; CAVAlCANTE, M. L. T M. H.; FORTES, M. M. P.; NEVES, P. P. \& ROCHA, A. C. K. "Os trigonum" y "Proceso de Stieda" en el síndrome del impacto posterior del tobillo. Int. J. Morphol., 31(4):1223-1226, 2013.

\section{REFERENCIAS BIBLIOGRÁFICAS}

Adams, J. C. \& Hamblen, D. L. Manual de Ortopedia. $11^{\text {a }}$ ed. Porto Alegre, Artes Médicas, 2010.

Bergman, R. A. \& Thompson, S. A. Catalog of Human Variation. Baltimore, Urban \& Schwarzenberg, 1984.

Chiereghin, A.; Martins, M. R.; Gomes, C. M. F.; Rosa, R. F.; Loduca, S. M. A. A. \& Chahade, W. H. Síndrome do Impacto Posterior do Tornozelo: um diagnóstico que deve ser lembrado pelo reumatologista: Relato de dois casos. Rev. Bras. Reumatol., 51(3):286-8, 2011.

Lima, C. M. A. O.; Ribeiro, E. B.; Coutinho, E. P. D.; Vianna, E. M.; Domingues, R. C. \& Júnior, A. C. C. Síndrome do impacto do tornozelo na ressonância magnética: ensaio iconográfico. Radiol. Bras., 43(1):53-7, 2010.

Llorca, F. O. Anatomía Humana. $3^{\mathrm{a}}$ ed. Tomo 1. Barcelona, Editorial Científico-Médica, 1967.

Moore, K. L.; Dalley, A. F. \& Agur, A. M. R. Anatomia Orientada para a Clínica. $6^{\mathrm{a}}$ ed. Rio de Janeiro, Guanabara Koogan, 2011.

Poirier, P. \& Charpy, A. Traité D’Anatomie Humaine. 9a ed. Vol.1. Paris, Masson et Cie. Editeurs, 1911.

Testut, L. \& Latarjet, A. Tratado de Anatomía Humana. Vol. 1. Barcelona, Salvat, 1959.

Thompson, J. C. Atlas de Anatomia Ortopédica de Netter. Porto Alegre, Artmed, 2004.

Xhardez, Y. Vade-mécum de Cinesioterapia. $4^{\mathrm{a}}$ ed. São Paulo, Andrei, 2001.
Dirección para Correspondencia:

Rua Teodoro da Silva, n491, apart. 101

Vila Isabel

Rio de Janeiro - RJ

CEP: 20.560-000.

BRASIL

Email: havasc@gmail.com.br meylie_tan@yahoo.com.br

Recibido : 13-07-2012

Aceptado: 22-08-2013 